\title{
microRNA-29b is a novel mediator of Sox2 function in the regulation of somatic cell reprogramming
}

Xudong Guo ${ }^{1, *}$, Qidong Liu ${ }^{1, *}$, Guiying Wang ${ }^{1}$, Songcheng Zhu ${ }^{1}$, Longfei Gao ${ }^{1}$, Wujun Hong ${ }^{1}$, Yafang Chen ${ }^{1}$, Minjuan $\mathrm{Wu}^{2}$, Houqi $\mathrm{Liu}^{2}$, Cizhong Jiang ${ }^{1}$, Jiuhong Kang ${ }^{1}$

${ }^{I}$ Clinical and Translational Research Center of Shanghai First Maternity \& Infant Health Hospital, Shanghai Key Laboratory of Signaling and Disease Research, School of Life Science and Technology, Tongji University, 1239 Siping Road, Shanghai 200092, China; ${ }^{2}$ Department of Embryology and Histology, Second Military Medical University, Shanghai 200433, China

Fibroblasts can be reprogrammed into induced pluripotent stem cells (iPSCs) by the application of Yamanaka factors (OSKM), but the mechanisms underlying this reprogramming remain poorly understood. Here, we report that Sox 2 directly regulates endogenous microRNA-29b (miR-29b) expression during iPSC generation and that miR-29b expression is required for OSKM- and OSK-mediated reprogramming. Mechanistic studies show that Dnmt3a and Dnmt3b are in vivo targets of miR-29b and that Dnmt3a and Dnmt3b expression is inversely correlated with miR-29b expression during reprogramming. Moreover, the effect of miR-29b on reprogramming can be blocked by Dnmt3a or Dnmt3b overexpression. Further experiments indicate that miR-29b-DNMT signaling is significantly involved in the regulation of DNA methylation-related reprogramming events, such as mesenchymal-to-epithelial transition (MET) and Dlk1-Dio3 region transcription. Thus, our studies not only reveal that miR-29b is a novel mediator of reprogramming factor Sox 2 but also provide evidence for a multistep mechanism in which Sox2 drives a miR-29b-DNMT signaling axis that regulates DNA methylation-related events during reprogramming.

Keywords: Sox2; miR-29b; Dnmt; reprogramming; MET; Dlk1-Dio3

Cell Research (2013) 23:142-156. doi:10.1038/cr.2012.180; published online 25 December 2012

\section{Introduction}

Direct reprogramming of somatic cells using the four transcription factors Oct4, Sox2, Klf4, and c-Myc (OSKM) has been employed to convert mouse and human somatic cells to an embryonic stem cell (ESC)like pluripotent state [1-6]. Fully reprogrammed induced pluripotent stem cells (iPSCs) are similar to ES cells in terms of their morphology, pluripotency gene expression pattern and ability to form teratomas with all three germ layers. Recently, germline transmission-competent chimeric mice and all-iPSC mice produced via tetraploid complementation have also been reported [3, 7-9]. The

\footnotetext{
*These two authors contributed equally to this work.

Correspondence: Jiuhong Kang

Tel: +086-21-65988876; Fax: +086-21-65981041

E-mail: jhkang@tongii.edu.cn

Received 29 May 2012; revised 7 September 2012; accepted 23 November 2012; published online 25 December 2012
}

iPSC technology thus provides a valuable tool for the study of human diseases and holds great potential in cellbased personalized therapy $[10,11]$. Among the OSKM factors, Oct4 and Sox 2 have been shown to be crucial for cell reprogramming $[12,13]$. However, the precise mechanisms by which these factors promote reprogramming remain poorly understood.

During iPSC generation, changes in DNA methylation are crucial for epigenetic remodeling and the reestablishment of the ESC-specific gene expression pattern [14]. Treatment of cells with 5-Aza-2'-deoxycytidine (AZA, a non-specific inhibitor of DNMTs) facilitates the transition from somatic cells to pluripotent stem cells [14-16], and DNA demethylation is required for the reactivation of epithelial genes in the MET process at the early stage of iPSC generation [17]. Recent studies have found that the activation of certain imprinted regions, such as the Dlk1-Dio3 locus, is correlated with the developmental potential of fully pluripotent iPSCs $[18,19]$. Aberrant DNA hypermethylation might be the major cause of the Dlk1-Dio3 region silencing that prevents cells from be- 
coming fully pluripotent iPSCs $[18,20]$. DNMTs have thus been considered barriers to reprogramming. However, DNMT expression is up-regulated at the late stage of iPSC generation, and it is required for the developmental potential of fully pluripotent iPSCs [21]. Moreover, conditional Dnmt3a or Dnmt3b knockout ES cells failed to differentiate into three germ layers [22]. These data indicate that DNMTs play different roles at different stages of cell reprogramming and that their expression is likely to be tightly controlled during reprogramming. However, how OSKM factors regulate the expression of DNMTs remains unclear.

MicroRNAs (miRNAs) are 20 25 nucleotide (nt) noncoding RNAs that generally bind to the $3^{\prime}$ untranslated regions (3'UTRs) of their target mRNAs through an imperfect match to suppress the translation and stability of these mRNAs [23]. An increasing number of studies have demonstrated that miRNAs specific to embryonic stem cells enhance the production of iPSCs [24]. Moreover, ectopic expression of the miR-302/367 cluster together with the knockdown of HDAC2 was shown to efficiently reprogram mouse and human somatic cells into iPSCs without requiring exogenous transcription factors [25], and a combination of mature miRNAs (miR-200c, miR-302s, and miR-369s) can also reprogram mouse and human cells into the pluripotent state [26]. Recent studies have revealed that both Oct4 and Sox 2 are involved in the regulation of miR-302 expression in hESCs [27, 28]; moreover, stimulating miR-302 expression in somatic cell reprogramming causes DNMT1 deficiency and global DNA hypomethylation [29]. These results indicate that miRNAs may serve as important mediators and play major roles in epigenetic and gene expression remodeling during OSKM-triggered iPSC generation.

Previous studies found that miR-29b mainly targets Dnmt $3 \mathrm{a} / 3 \mathrm{~b}$ and functions in cancer development [30, 31]. miR-29b has been shown to induce global DNA hypomethylation and the re-expression of specific genes, such as $\mathrm{p} 15^{\mathrm{INK} 4 \mathrm{~b}}$, in human acute myeloid leukemia (AML) cells [30] and to regulate genomic DNA methylation in lung cancer and mouse primordial germ cells by targeting Dnmt3a and Dnmt3b [31, 32]. However, it remains unknown whether miR-29b is involved in the regulation of DNA methylation remodeling during iPSC generation and how this miRNA mediates OSKM-triggered cell reprogramming.

By studying miRNAs that are specifically increased on the indicated days of iPSC induction, we found that miR-29b is directly upregulated by Sox 2 and required for OSKM- and OSK-triggered somatic cell reprogramming. Target gene analysis showed that miR-29b inhibits the expression of Dnmt3a/3b. Dnmt3a/3b expression is inversely correlated with miR-29b expression during reprogramming. Further, we demonstrated that miR-29b is a crucial mediator that helps Sox 2 control the dynamic expression of Dnmt3a/3b during iPSC generation.

\section{Results}

miR-29b is upregulated by Sox 2 and is involved in iPSC induction

According to our microarray data, the expression of miR-29b was significantly increased during OSKM induction. The expression profile of $\mathrm{miR}-29 \mathrm{a} / \mathrm{c}$, which belongs to the miR-29b family, was similar to that of miR-29b after OSKM induction, but the expression level of miR-29b was more significantly changed than that of miR-29a/c during the reprogramming process (Supplementary information, Figure S1A). To investigate the contributions of the defined factors to the increased expression of miR-29b, we used bioinformatics prediction software to analyze the potential transcription factor binding sites within the miR-29b promoter. We found that this region contains a potential Sox 2 binding site but no putative Oct4, Klf4 or c-Myc binding sites. Then, we expressed Sox 2 and Oct4 individually in Oct4::GFP MEFs (OG-MEFs) and observed that Sox2, but not Oct4, stimulated a $\sim 2.3$-fold increase in miR-29b expression (Figure 1A). Furthermore, the presence of the Sox2 binding site in the miR-29b promoter was confirmed by chromatin immunoprecipitation (ChIP) analysis (Figure 1B) and luciferase reporter assay (Figure 1C). We next studied whether miR-29b acts downstream of Sox 2 to enhance iPSC generation. For this purpose, we constructed two retroviral vectors to produce the mature miR-29b or the miR-29b sponge, respectively (Supplementary information, Figure S1B). The numbers of alkaline phosphatase positive $\left(\mathrm{AP}^{+}\right)$and Oct4::GFP positive $\left(\mathrm{GFP}^{+}\right)$colonies upon iPSC induction were significantly increased when miR-29b was included in the OSKM or OSK iPSC induction than when OSKM or OSK were used alone. In contrast, compared to OSKM- or OSK-mediated reprogramming, the introduction of the miR-29b sponge obviously decreased the efficiency of $\mathrm{AP}^{+}$and $\mathrm{GFP}^{+}$colony formation (Figure 1D and 1E), indicating that miR-29b likely serves as a direct mediator of Sox 2 and plays a positive role in somatic cell reprogramming.

The pluripotency and developmental potential of miR29b-derived iPSCs

To examine the pluripotency of OSKM + miR-29bderived iPSCs, we first checked the activity of Oct 4 and alkaline phosphatase. The results showed that all iPSC colonies were both $\mathrm{GFP}^{+}$and $\mathrm{AP}^{+}$(Figure 2A). Pluripo- 
A

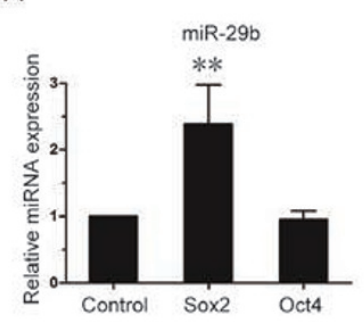

D

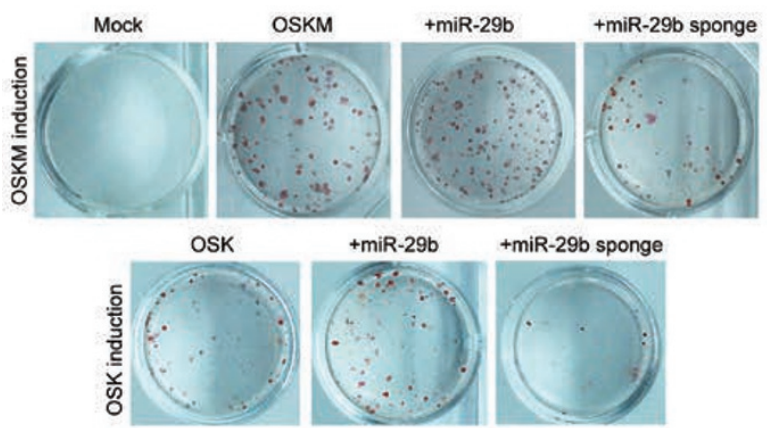

E

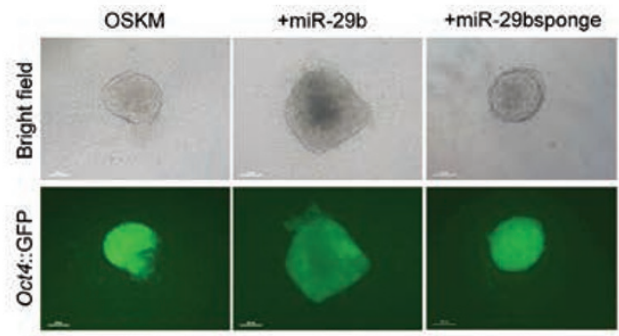

OSK

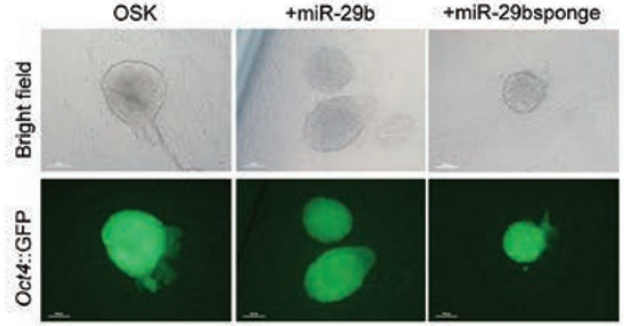

C
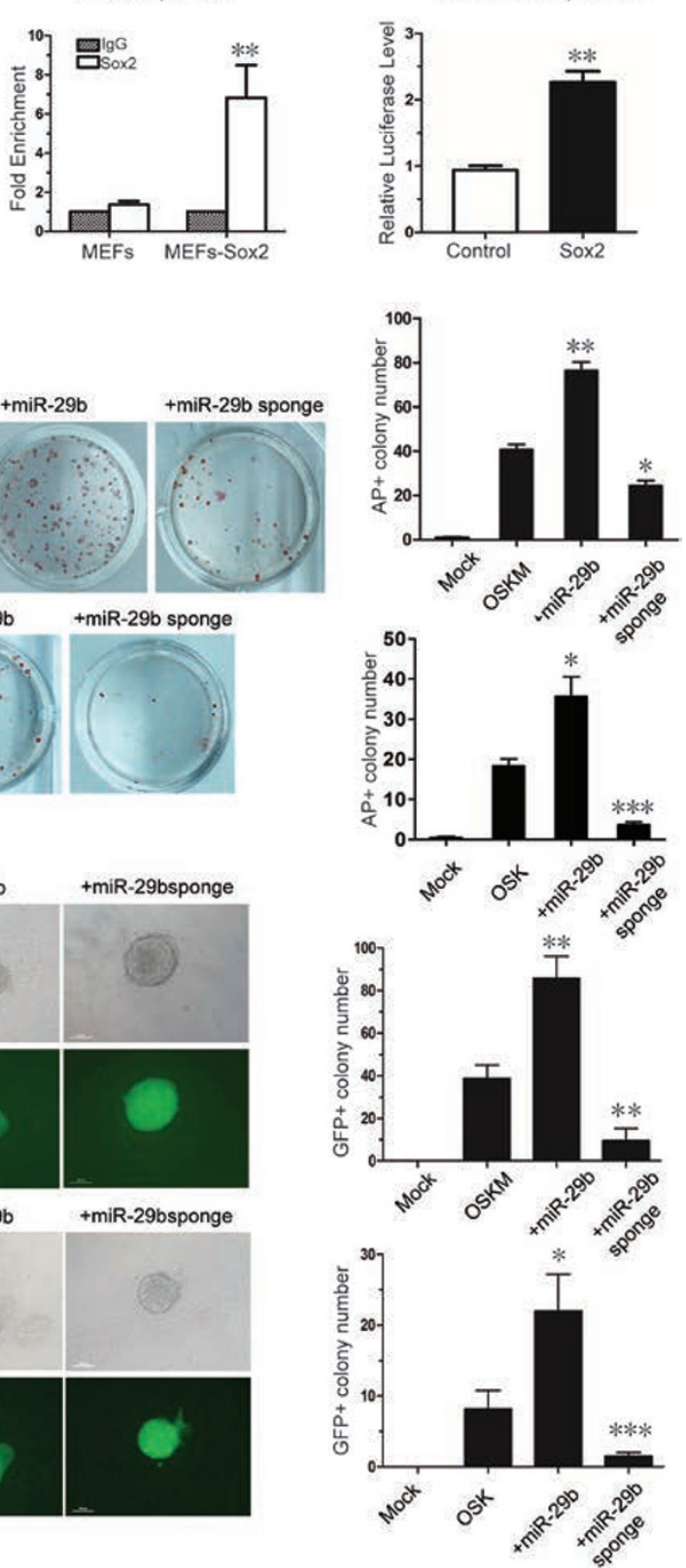

Figure 1 miR-29b is upregulated by Sox2 and is involved in iPSC induction. (A) The effects of retroviruses containing Sox2 or Oct4 on miR-29b expression in OG-MEFs. (B) The fold enrichment of Sox2 binding at the miR-29b promoter regions. ChIP analyses using anti-Sox2 antibody and normal rabbit IgG were performed with OG-MEFs or Sox2-introduced OG-MEFs (MEFs-Sox2). The fold enrichment of Sox2 binding was normalized to the level of IgG binding. (C) Luciferase reporter assays were performed with vectors containing DNA fragments of the miR-29b promoter, which contains a Sox2 binding site. The luciferase reporter vector was co-transfected with the pMX-Sox2 (Sox2) or pMX-GFP (Control) vector into NIH3T3 cells. Firefly luciferase activity was normalized to Renilla luciferase activity. (D) Representative images of $\mathrm{AP}^{+}$colonies (left) and the quantification of $\mathrm{AP}^{+}$colonies (right) on day 10 after OSKM retroviral infection (day 12 for OSK transduction). OG-MEFs were infected with retroviruses containing OSKM or OSK in combination with miR-29b or the miR-29b sponge, respectively. Cultures were fixed and stained for alkaline phosphatase activity. (E) The morphology of typical Oct4::GFP iPSC colonies and quantification of $\mathrm{GFP}^{+}$colony numbers on day 12 after OSKM retroviral infection (day 16 for OSK transduction). Representative phase contrast images and fluorescence images are shown (left). As a more stringent quantification of reprogramming efficiency, GFP ${ }^{+}$colony numbers were counted under a fluorescence microscope (right). Error bars represent the standard deviation (SD) of three independent experiments. ${ }^{*} P<0.05,{ }^{* *} P<0.01$, two-tailed Student's $t$-test. 
A

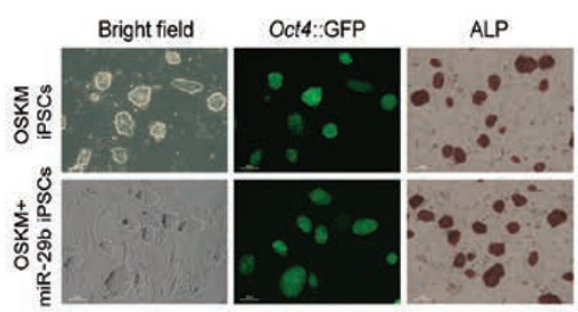

C

D

E

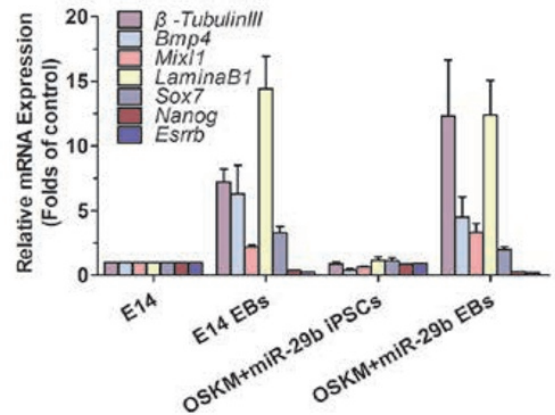

B
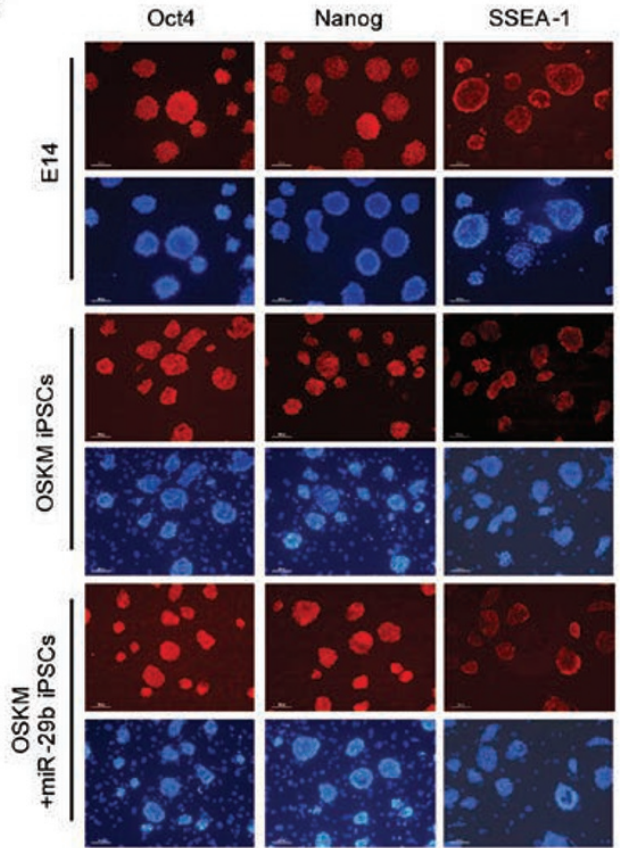

G
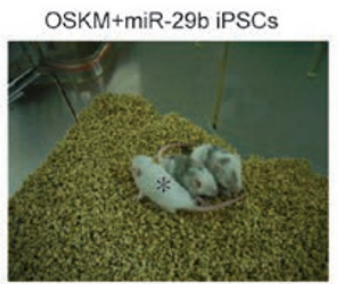

F
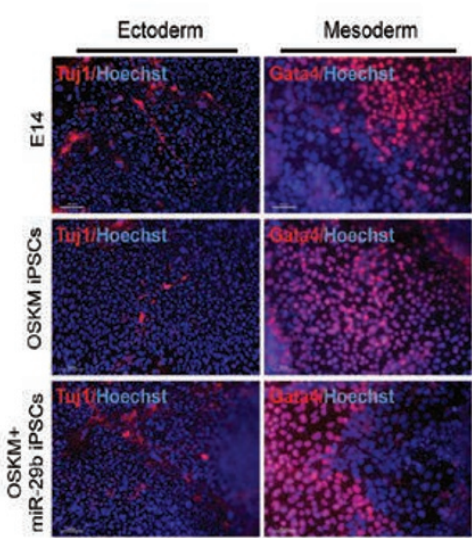
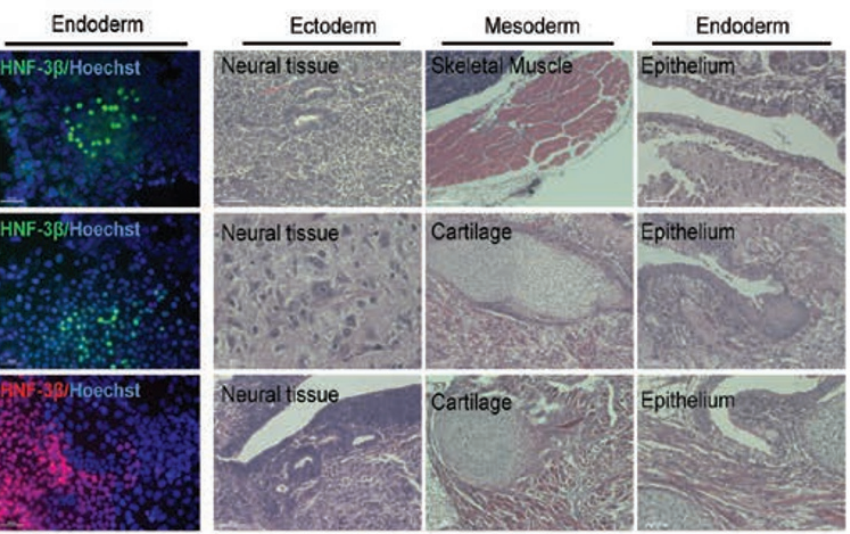

Figure 2 Pluripotency analyses and the developmental potentials of miR-29b-derived iPSCs. (A) OSKM+miR-29b-derived iPSC colonies exhibited typical stem cell morphology, expressed Oct4::GFP and exhibited high alkaline phosphatase activity. (B) OSKM iPSCs and OSKM + miR-29b-derived iPSCs expressed mouse pluripotency markers (Oct4, Nanog, and SSEA1), similar to E14 mouse embryonic stem cells. (C) RT-PCR analyses of endogenous Oct4, Sox2, KIf4, c-Myc, and Nanog expression. Gapdh was used as an internal control. (D) QRT-PCR analyses of markers of all three germ layers and pluripotency factors in iPSCs and EBs. The error bars represent the expression level relative to that of E14. Gapdh was used as an internal control. (E) Immunostaining shows that OSKM iPSCs and OSKM+miR-29b-derived iPSCs can differentiate into cells expressing characteristic markers of the three germ layers, similar to E14 cells. (F) Teratomas derived from E14 cells, OSKM iPSCs, and OSKM+miR-29b-derived iPSCs. Representative images of HE staining for neural tissue (ectoderm), cartilage or skeletal muscle (mesoderm), and epithelial tissue (endoderm) are shown. (G) Two-week-old chimeric mice derived from OSKM+miR-29b-derived iPSCs (C57BL/6 background). Asterisk indicates control ICR mice. 
tency marker expression was also confirmed by immunostaining (Oct4, Nanog, and SSEA1, Figure 2B), RTPCR (endogenous Oct4, Sox2, Klf4, c-Myc, and Nanog, Figure 2C), and QRT-PCR (Nanog, Esrrb, Utf1, and Dppa5, Supplementary information, Figure S2A). Exogenous transgenes were silenced in the OSKM + miR-29b-derived iPSC lines (Supplementary information, Figure S2B). Analyses of the genetic integration of OSKM factors and miR-29b retrovirus insertion showed that the colonies were derived from cells that had been infected by OSKM and miR-29b simultaneously (Supplementary information, Figure S2C). Consistent with the activation of endogenous Oct4 and Nanog, the DNA methylation level in the promoter regions of these genes was lower in OSKM + miR-29b-derived iPSCs than in OG-MEFs (Supplementary information, Figure S2D). Therefore, the iPSCs produced by transduction with OSKM and miR$29 \mathrm{~b}$ are pluripotent with respect to the activation of pluripotency genes.

To investigate whether the derived colonies exhibit the full differentiation potentials of ESCs, we used floating cultivation to form embryoid bodies (EBs). After 3 days in suspension culture, the miR-29b-derived colonies developed into ball-shaped structures, exhibiting efficient EB formation (Supplementary information, Figure S3A). Then, we transferred these EBs to gelatin-coated plates and continued their cultivation for 6 days (Supplementary information, Figure S3A). Immunostaining showed that the EBs generated from the OSKM + miR-29b-derived iPSCs expressed lineage markers such as Tuj1 (ectoderm), Gata4 (mesoderm), and HNF-3 $\beta$ (endoderm) (Figure 2E). QRT-PCR confirmed that these differentiated EBs expressed relevant markers for all three germ layers (Figure 2D and Supplementary information, Figure S3B). In contrast, the expression of pluripotency markers such as Nanog and Esrrb was markedly decreased (Figure 2D). Further, the OSKM + miR-29b-derived iPSCs were injected into the dorsal flanks of athymic nude mice (NODSCID) to test their ability to form teratomas. E14 cells were also injected as a control. Teratomas were readily observed at 4 weeks after iPSC injection, and the subsequent histological analysis showed that the tumors generated by the OSKM + miR-29b-derived iPSCs contained neural tissue (ectoderm), skeletal muscle (mesoderm), cartilage (mesoderm), and epithelial tissue (endoderm) (Figure 2F). To further demonstrate the pluripotency of OSKM + miR-29b-derived iPSCs, we performed chimera generation to test their ability to generate chimeric mice. Live chimeras with major contributions from OSKM + miR-29b-derived iPSCs were obtained, demonstrating the in vivo differentiation potential of these iPS cells (Figure $2 \mathrm{G}$ ). Taken together, our results showed that the iPS cells generated by OSKM plus miR-29b are fully pluripotent iPS cells.

\section{miR-29b specifically represses Dnmt3a and Dnmt3b ex-} pression

To further understand the mechanism underlying the effect of miR-29b on reprogramming, we investigated the targets of miR-29b. Based on the previous studies [30-32] and in silico analyses using target prediction programs including TargetScan, Miranda and miRBase, we predicted that Dnmt3a and Dnmt3b might be targeted by miR-29b during reprogramming (Figure $3 \mathrm{~A}$ ). Then, we performed luciferase transcription assays using the Dn$\mathrm{mt3a}$ and Dnmt3b wild-type 3'UTR luciferase reporters. A luciferase reporter containing the Oct4-3'UTR, which was predicted to be resistant to miR-29b both by in silico analyses and by our previous observations, was used as a negative control. As shown in Figure 3B, we found that Dnmt3a and Dnmt3b wild-type 3'UTR luciferase reporter activities were significantly repressed by the application of miR-29b mimics compared to control mimics at $24 \mathrm{~h}$ post-transfection. The regulation of Dnmt3a and Dnmt3b by miR-29b was further confirmed by the observation that miR-29b had little effect on the expression of Dnmt3a and Dnmt3b luciferase constructs with mutant 3'UTR sequences. Dnmt3a and Dnmt3b luciferase reporter activities were also inhibited by endogenous miR29b in NIH3T3 cells relative to Oct4-3'UTR luciferase activities (Figure 3B). Consistent with these observations, the endogenous expression of Dnmt3a and Dnmt3b was significantly inhibited by miR-29b mimics and the miR-29b retrovirus but enhanced by a miR-29b inhibitor and the miR-29b sponge (Figure 3C). These results indicate that Dnmt3a and Dnmt3b are in vivo targets of miR$29 b$.

\section{Dnmt3a and Dnmt3b significantly block iPSC generation}

The DNA methylation levels of the promoters of many pluripotency factors, such as Oct4 and Nanog, have been reported to be correlated with cell reprogramming [35]. Dnmt3a and Dnmt3b are enzymes that control de novo DNA methylation. Here, we examined the effects of Dnmt3a and Dnmt3b on reprogramming efficiency. The efficiency of Dnmt3a and Dnmt3b overexpression and knockdown was determined by western blotting analyses (Figure 4A and 4B). The efficiency of iPSC generation by OSKM factors was greatly decreased when Dnmt3a or Dnmt3b was overexpressed in OG-MEFs as demonstrated by the number of $\mathrm{AP}^{+}$colonies (Figure 4D). The representative images of $\mathrm{AP}^{+}$colonies are shown in Figure 4C. Consistently, the introduction of Dnmt3a and Dnmt3b resulted in a lower efficiency of $\mathrm{GFP}^{+}$colony 
A

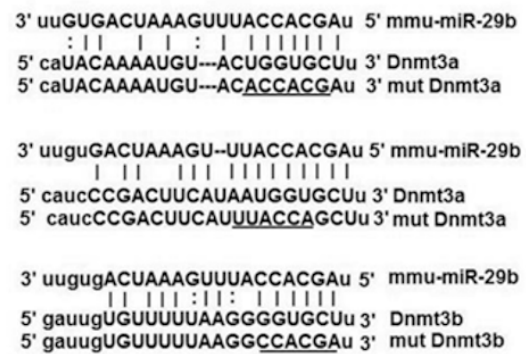

B
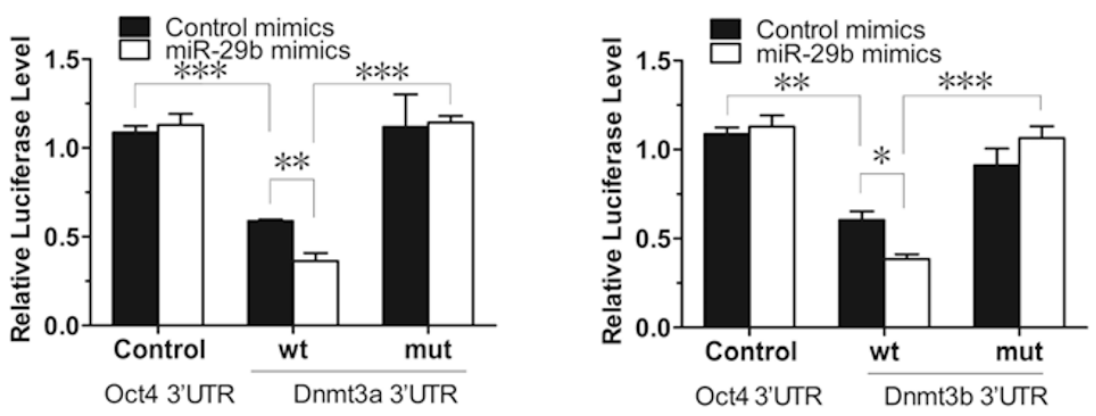

C

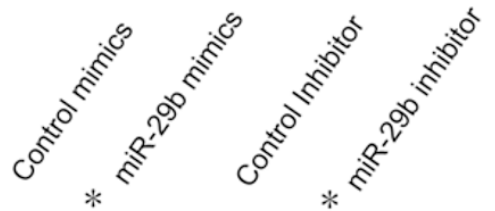
$\begin{array}{llll}1.00 & 0.42 \pm 0.08 & 1.00 & 3.39\end{array} 0.99$
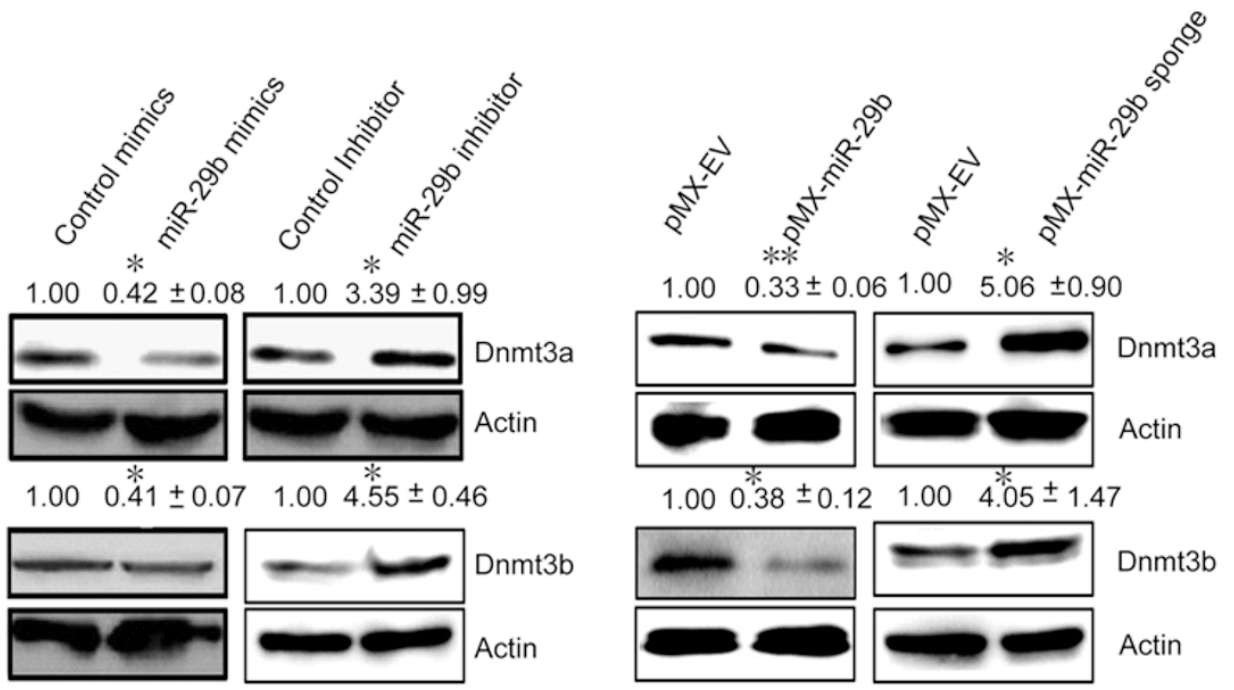

Figure 3 Dnmt3a and Dnmt3b are the in vivo targets of miR-29b. (A) miR-29b target sites in the $3^{\prime} U T R s$ of Dnmt3a and Dnmt3b mRNA are shown, aligned with the miR-29b sequence. (B) Luciferase reporter assays were performed with vectors containing DNA fragments corresponding to the putative wild-type or mutant target sites for miR-29b in the $3^{\prime} U T R$ s of the Dnmt3a and Dnmt3b mRNAs. The luciferase reporter vector was co-transfected with miR-29b mimics or control mimics into NIH3T3 cells. Firefly luciferase activity was normalized to Renilla luciferase activity. The vector containing the $3^{\prime} U T R$ of Oct4 was used as a negative control in the assays, and the data shown indicate the means $\pm S D$ of three independent experiments. ${ }^{*} P<0.05,{ }^{* *} P<0.01,{ }^{* * *} P<0.001$, two-tailed Student's $t$-test. (C) Western blotting analyses of Dnmt3a and Dnmt3b in MEFs after transfection with miR-29b mimics or miR-29b inhibitors (left) and transduction with miR-29b or miR-29b sponge (Right). pMX-EV (empty vector), control mimics and control inhibitors were used as negative controls. Actin was used as a loading control (lower panel). For quantification, relative signal intensity determined by densitometric analysis is shown. The

formation (Figure 4E and 4F). In contrast, Dnmt3a and Dnmt3b knockdown using shRNAs increased the number of both $\mathrm{AP}^{+}$and $\mathrm{GFP}^{+}$colonies (Figure 4D and 4F). These results indicate that Dnmt3a and Dnmt3b may serve as a barrier to reprogramming at the early stage and that their knockdown facilitates reprogramming.

miR-29b enhances iPSC formation via targeting Dnmt3a 
A

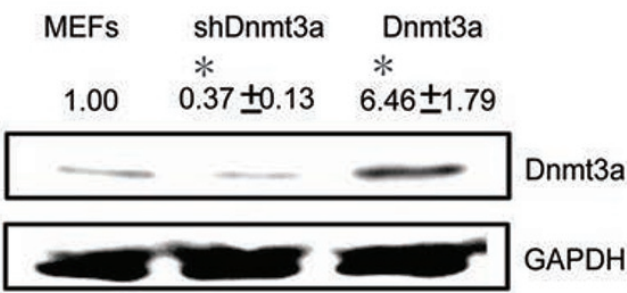

B

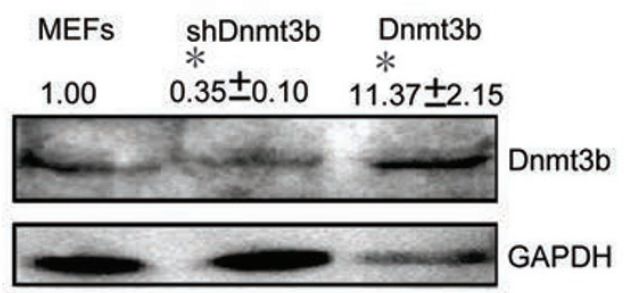

E

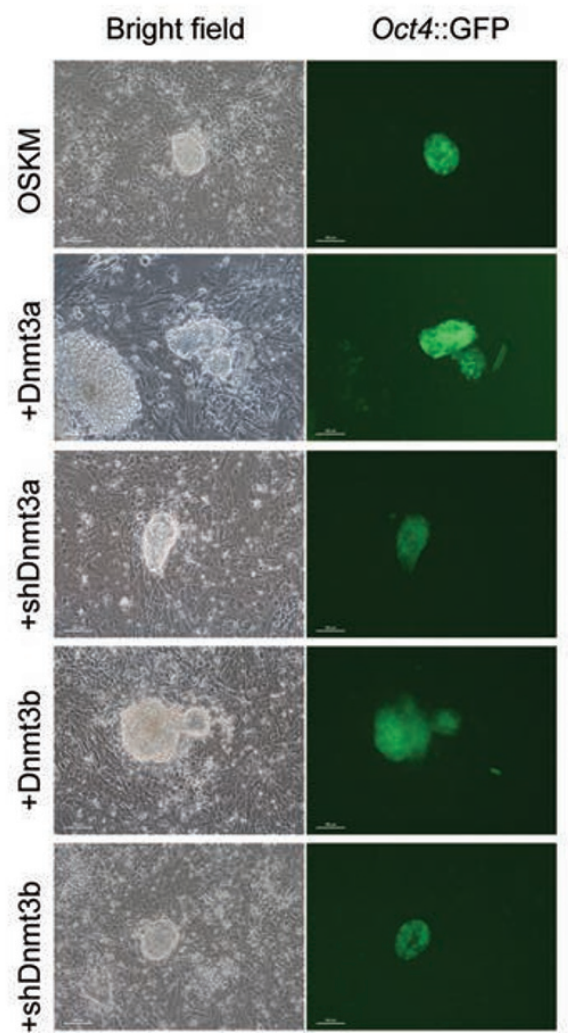

C

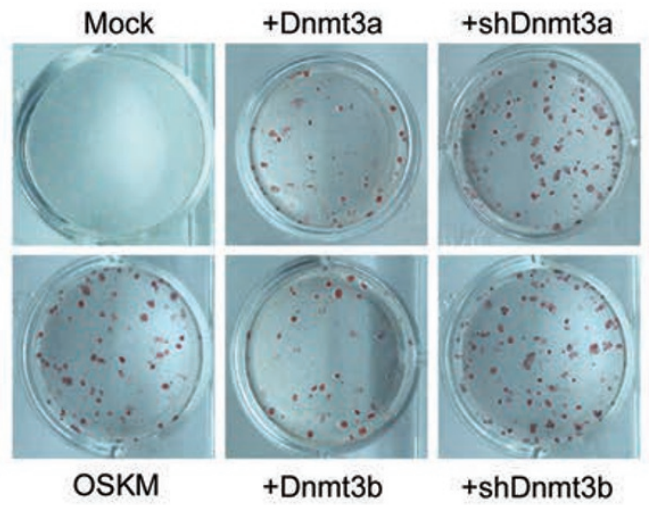

D

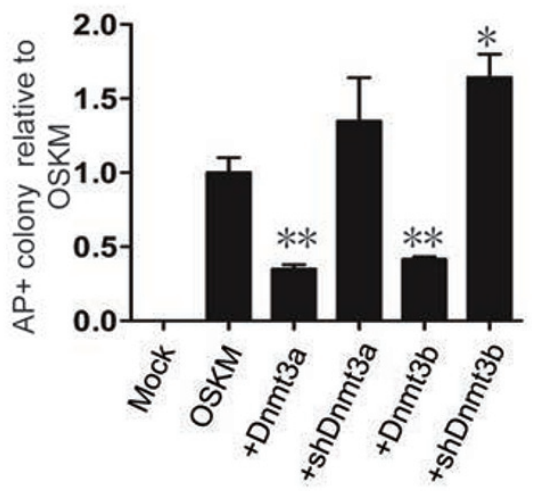

F

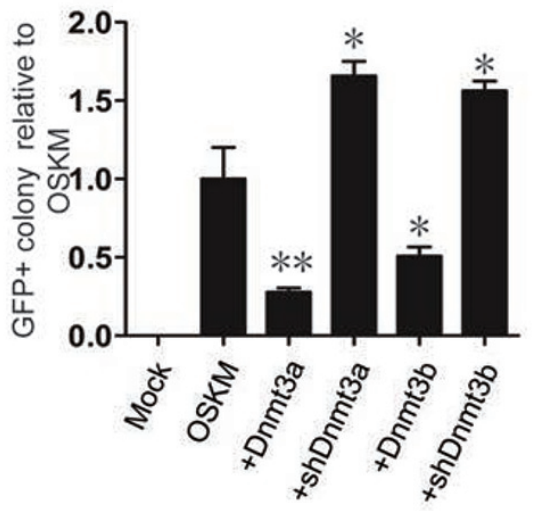

Figure 4 Dnmt3a and Dnmt3b inhibit iPSC generation. (A, B) Transduced MEFs were tested for the over-expression and knockdown efficiency of Dnmt3a or Dnmt3b by western blotting. (C, D) Images and quantification of $\mathrm{AP}^{+}$colonies on day 10 after OSKM transduction in combination with Dnmt3a, Dnmt3b, Dnmt3a shRNA (shDnmt3a), or Dnmt3b shRNA (shDnmt3b). $(E, F)$ Representative images and quantification of GFP $^{+}$colonies on day 12 after OSKM transduction in combination with Dnmt3a, Dnmt3b, shDnmt3a, or shDnmt3b. Error bars represent the SD of three independent experiments. ${ }^{*} P<0.05$, ${ }^{* *} P<$ 0.01 , two-tailed Student's $t$-test.

and Dnmt3b

We also used QRT-PCR to show that the expression of miR-29b was increased after OSKM were ectopically expressed in OG-MEFs (Figure 5A). The expression of Dnmt3a and Dnmt3b was downregulated as miR-29b increased at the early stage of OSKM induction, indicating 
A

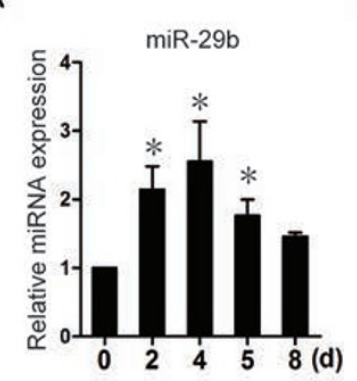

B

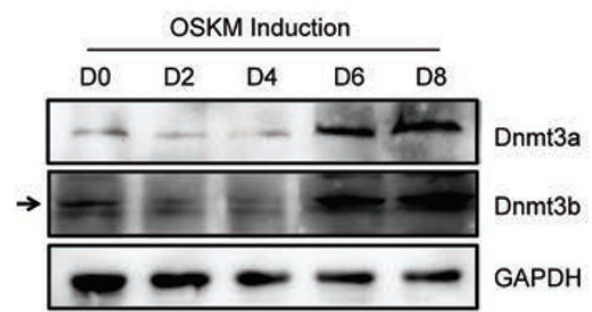

C

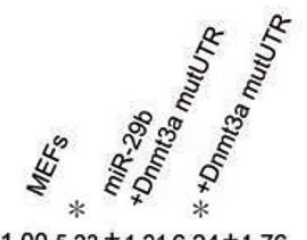

$1.005 .23 \pm 1.216 .24 \pm 1.76$

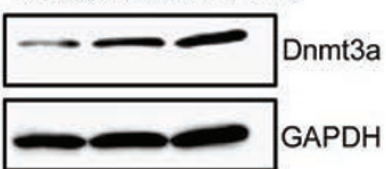

D

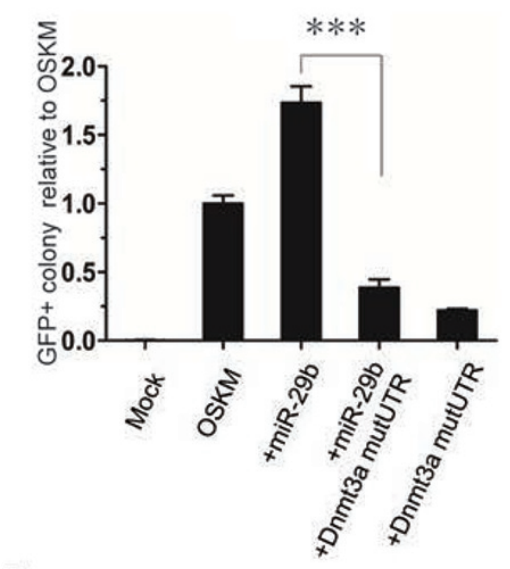

E

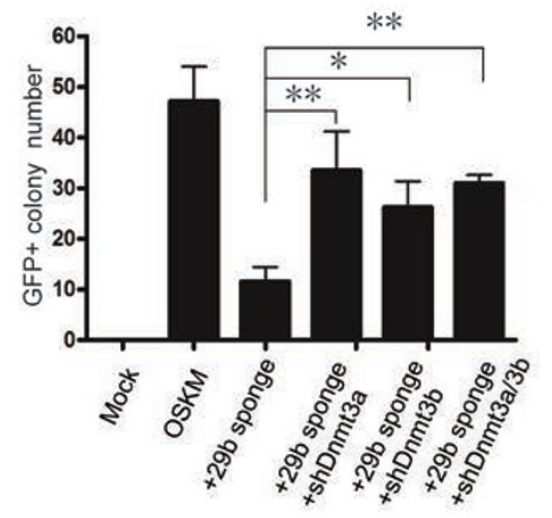

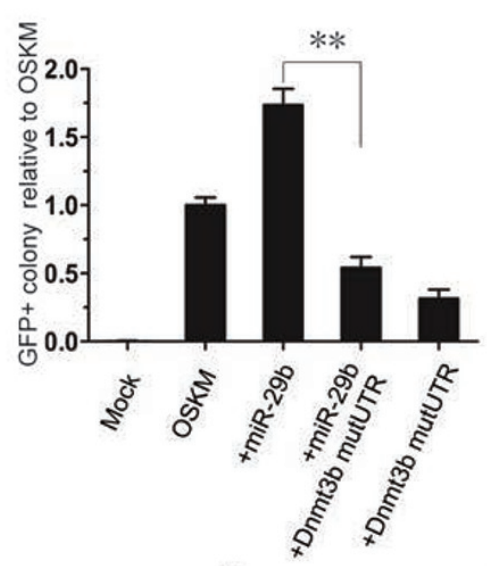

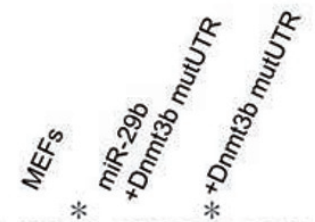

$1.007 .23 \pm 2.087 .54 \pm 2.61$
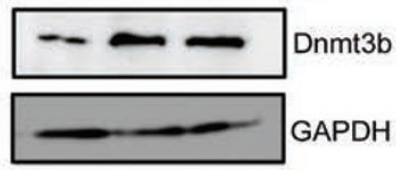

$\mathrm{F}$
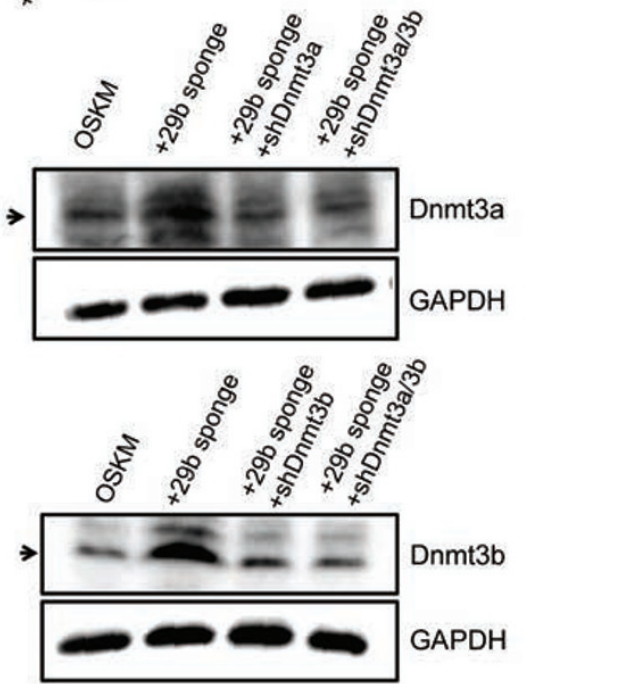

Figure 5 miR-29b enhances iPSC formation by targeting Dnmt3a and Dnmt3b. (A) QRT-PCR analysis of miR-29b expression on the indicated days in OSKM transduced OG-MEFs. U6 was used as an internal control. (B) Western blotting analyses of Dnmt3a/b protein expression during OSKM induction. (C) Western blot analyses of Dnmt3a and Dnmt3b expression in MEFs co-transduced with miR-29b mimics and Dnmt3a-UTRmut or Dnmt3b-UTRmut. GAPDH was used as a loading control. The data shown indicate the means \pm SD of three independent experiments. (D) miR-29b induction efficiency was abrogated by the ectopic expression of Dnmt3a-UTRmut or Dnmt3b-UTRmut. OG-MEFs were co-transduced with retroviruses containing OSKM + miR-29b, OSKM + miR-29b + Dnmt3a/3b-UTRmut, or OSKM + Dnmt3a/3b-UTRmut and cultured under standard iPSC induction conditions. GFP ${ }^{+}$colonies were quantified on day 10 post-transduction. (E) The inhibition of induction efficiency by the miR-29b sponge was reversed by shDnmt3a or shDnmt3b. GFP ${ }^{+}$colonies were quantified on day 12 post-transduction. (F) Western blotting showing the expression of Dnmt3a or Dnmt3b during OSKM reprogramming after the introduction of the miR-29b sponge or a combination of the miR-29b sponge with shDnmt3a and/or shDnmt3b. The error bars represent the standard deviation (SD) of three independent experiments. ${ }^{*} P<0.05$, ${ }^{* *} P<0.01$, ${ }^{* * *} P<0.001$, two-tailed Student's $t$-test. 
that the expression of Dnmt3a and Dnmt3b is inversely correlated with that of miR-29b at the early stage of reprogramming (Figure 5B). To determine whether miR$29 \mathrm{~b}$ promotes reprogramming by repressing Dnmt3a and Dnmt3b expression, we conducted a series of rescue assays. In the presence of OSKM, we compared the reprogramming efficiencies of adding miR-29b, Dnmt3aUTRmut, Dnmt3b-UTRmut, or a combination of miR29b with Dnmt3a-UTRmut or Dnmt3b-UTRmut. The results showed that the addition of miR-29b improved the reprogramming efficiency, while the combination of miR-29b with Dnmt3a-UTRmut or Dnmt3b-UTRmut blocked the effect of miR-29b on reprogramming (Figure 5D and Supplementary information, Figure S4A and S4B). MiR-29b had no effect on the expression of Dnmt3a-UTRmut or Dnmt3b-UTRmut (Figure 5C). Moreover, the combination of the miR-29b sponge with the Dnmt3a and/or Dnmt3b shRNAs reversed the inhibition of iPSC induction by miR-29b sponge (Figure 5E). During iPSC generation, the introduction of miR-29b sponge led to the upregulation of Dnmt3a and Dnmt3b expression (Figure 5F). These findings suggest that miR$29 \mathrm{~b}$ improved the reprogramming efficiency by targeting Dnmt3a and Dnmt3b.

miR-29b-DNMT signaling is involved in regulating MET and the transcriptional silencing of the Dlk1-Dio3 region.

Mesenchymal-to-epithelial transition (MET) is a DNA methylation-related event known to be required at the initial stage of cell reprogramming $[17,38]$. We tested whether the increased expression of miR-29b at the early stage of induction was associated with MET by introducing the OSKM factors into OG-MEFs with or without miR-29b. We found that the expression of epithelial genes such as E-cadherin, Epcam, and Cldn3 was significantly increased in the cells that were induced with a combination of OSKM and miR-29b compared to those that were induced with OSKM alone (Figure 6A). Consistent with this observation, western blotting analysis of E-cadherin protein expression showed that miR$29 \mathrm{~b}$ significantly activated the expression of E-cadherin during reprogramming (Figure 6B). In addition, we found that the expression of mesenchymal genes such as Cdh2, Snail, and Zeb1 was dramatically decreased in the OSKM plus miR-29b groups at different time points (day 4,6 , and 8 ) during reprogramming, further confirming that miR-29b promotes MET (Supplementary information, Figure S5B). The analysis of E-cadherin and Cdh2 promoter methylation on the indicated days of reprogramming (Figure 6C and Supplementary information, Figure S5A) supports that miR-29b was involved in the activation of E-cadherin and in MET. Moreover, the expression of the epithelial gene E-cadherin was increased when Dnmt3a or Dnmt3b was knocked down, whereas the expression of the mesenchymal genes Zeb1 and Snail was significantly decreased (Figure 6D). Consistently, the overexpression of Dnmt3a or Dnmt3b exerted opposite effects on the expression of these genes involved in MET (Figure 6D). These data showed that the miR-29b-DNMT signaling axis was significantly involved in the MET process. As miR-29b might be considered a mediator of Sox2-driven reprogramming, we tested whether miR$29 \mathrm{~b}$ could activate MET in the absence of Sox 2 . Our data showed that miR-29b enhanced the expression of epithelial genes such as E-cadherin, Epcam, and Cldn3 in the context of OKM induction as well as in OSKM induction (Supplementary information, Figure S5C). As a control, we showed that the expression of genes such as Cripto, Utf1, and Fgf4, which are increasingly transcribed at the later stage of reprogramming, was not affected by the expression of miR-29b (Supplementary information, Figure S5D). Overall, miR-29b-DNMT signaling is likely involved in regulating the early stage of MET to promote the reprogramming of MEFs to iPSCs.

Studies from Stadtfeld et al. [20] and Liu et al. [18] showed that the imprinted Dlk1-Dio3 region is mostly silenced by DNA methylation in OSKM-induced iPSCs and that the activation of this imprinted region might actually correlate with the degree of iPSC pluripotency. To probe the potential effect of miR-29b on the activation of the Dlk1-Dio3 region, we analyzed the expression of the genes and miRNAs in the Dlk1-Dio3 region in both OSKM-derived iPSC lines and OSKM + miR-29b-derived iPSC lines. We found that Gt12, Rian, and miRNAs (miR-409-5p, miR-127, miR-376c, and miR-136) were all more highly expressed in the OSKM + miR-29bderived iPSCs than in the OSKM-iPSC lines (Figure 6E and Supplementary information, Figure S6A), consistent with the results of the bisulfite sequencing analysis of CpG methylation at the differentially methylated region (DMR) of Gtl2 (Figure 6F). Similarly, the iPSC lines generated by OSK and miR-29b also showed greater Gt12, Rian and miRNA expression (Supplementary information, Figure S6B). We also investigated whether miR$29 \mathrm{~b}$ could prevent the silencing of the Dlk1-Dio3 locus during the reprogramming process. As expected, due to the low reprogramming efficiency $(\sim 0.1 \%)$ and background of MEFs, the methylation level of the Dlk1-Dio3 locus and the expression of genes Gt12 and Rian were not significantly changed at the indicated days after either OSKM or OSKM + miR-29b transduction (Supplementary information, Figure S6C and S6D). Interestingly, the addition of miR-29b mimics increased the expression of 
A
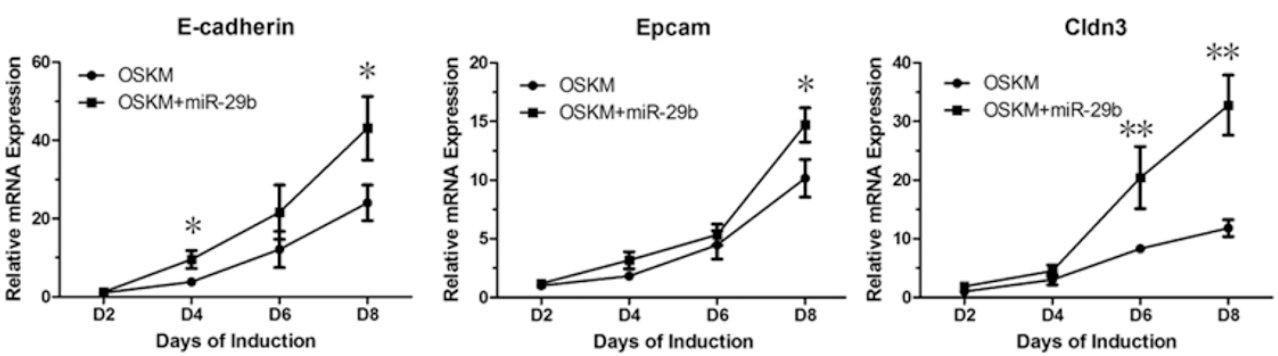

B

C
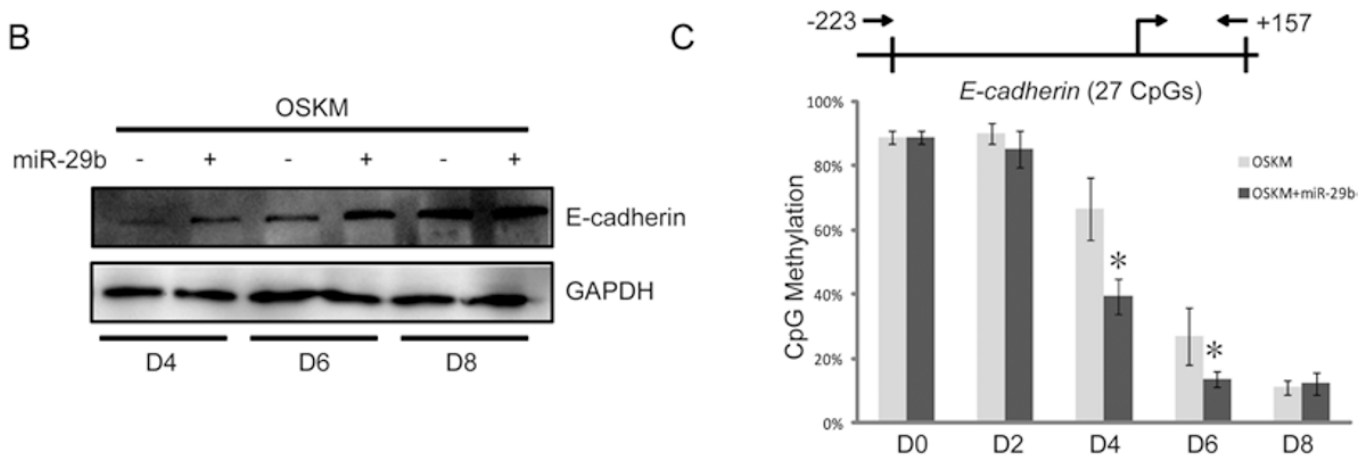

D
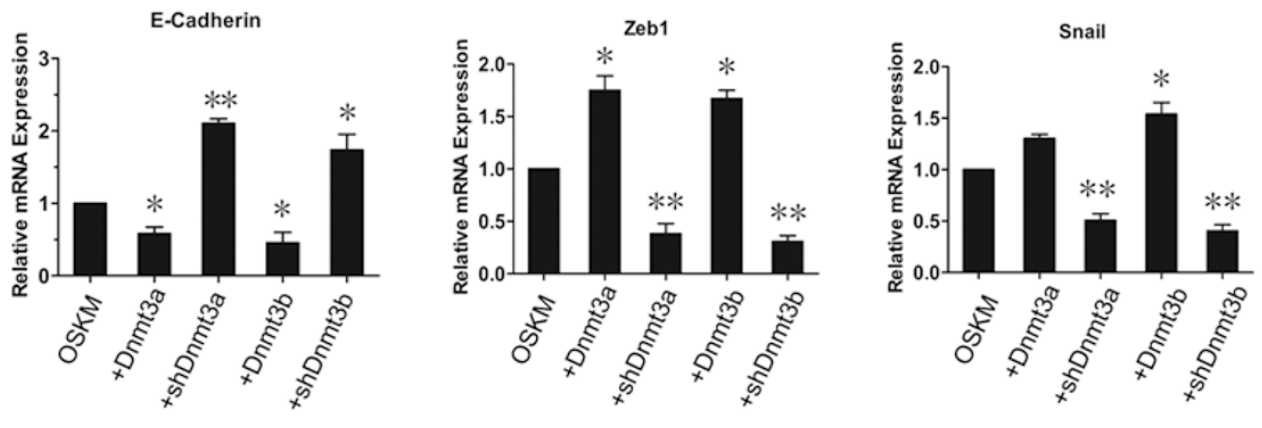

E
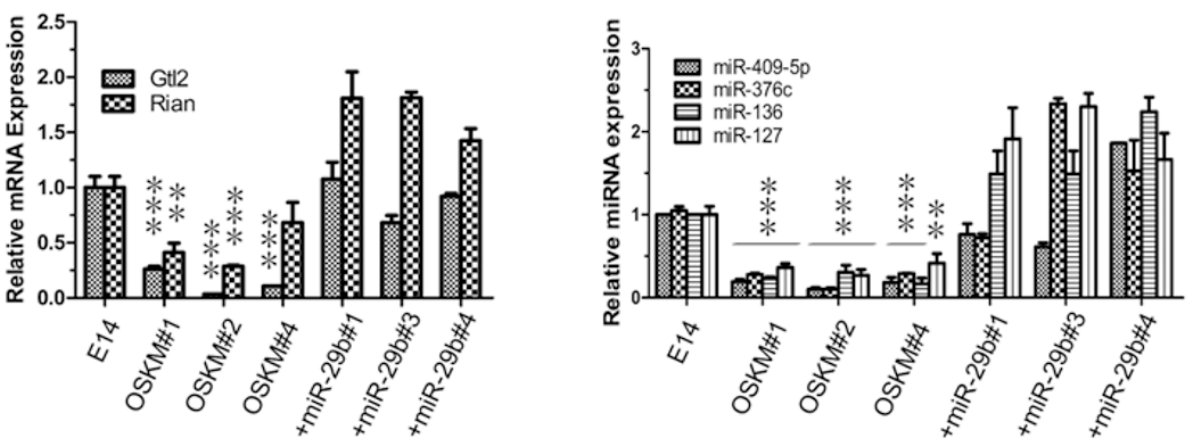

F

G
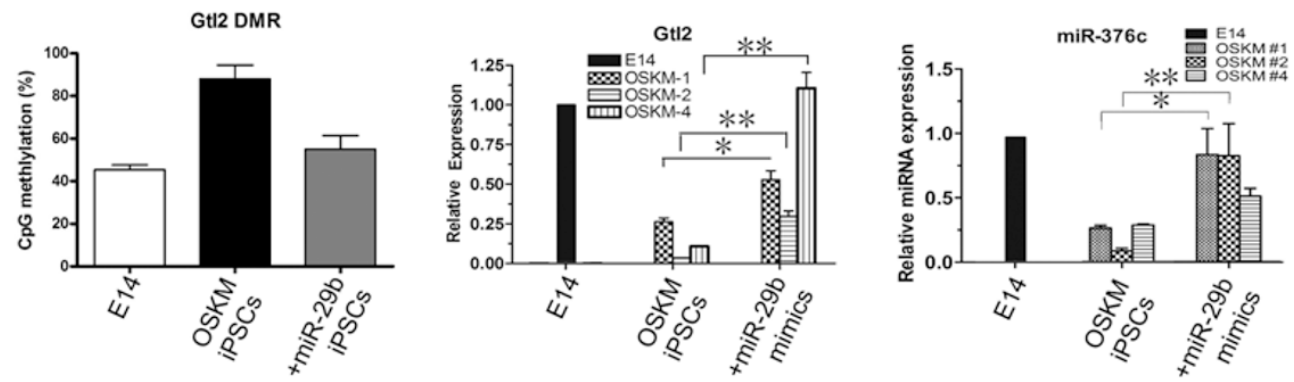
genes and miRNAs in the Dlk1-Dio3 locus at $72 \mathrm{~h}$ posttransfection in OSKM-iPSCs (Figure 6G and Supplementary information, Figure S6E). These data suggest that miR-29b-DNMT signaling plays important roles in regulating DNA methylation-related reprogramming events, including MET and Dlkl-Dio3 region silencing.

\section{Discussion}

miRNAs have been identified and implicated in the regulation of somatic cell reprogramming [24-26]. Previous studies showed that key transcription regulators such as Oct4, Sox2, and Nanog occupy the promoters of a set of miRNA genes (e.g., the miR-290 cluster and miR$302 / 367$ cluster). These miRNAs not only play important roles in the maintenance of ES cell pluripotency and self-renewal but also promote iPSC generation by suppressing the expression of their specific target genes [24, 27-29], suggesting that the direct regulation of miRNA expression is one important way for the defined factors to specifically regulate gene expression and cell reprogramming. In the present study, we found that the defined factor Sox 2 binds to the promoter region of miR-29b and regulates the transcription of endogenous miR-29b during iPSC induction. While blocking the function of endogenous miR-29b using miR-29b sponge significantly repressed OSKM-induced iPSC generation, transducing miR-29b along with OSKM significantly increased iPSC induction efficiency but did not reduce the pluripotency or development potential of iPSCs. These results not only indicate that miR-29b is a key target gene of Sox2 during somatic cell reprogramming but also demonstrate that facilitating cell reprogramming and pluripotency acqusition is a previously unrevealed function of miR-29b.

The rearrangement of DNA methylation patterns is essential for successful somatic cell reprogramming [36]. Dnmt3a and Dnmt3b are de novo methyltransferases that are critically important for the establishment of DNA methylation patterns during cell differentiation and reprogramming [37]. Treatment with AZA facilitates iPSC induction by three factors (Oct4, Sox2, and Klf4) or only two factors (Oct4 and Klf4) [14-16]. Consistent with these data, our study showed that the knockdown of either Dnmt3a or Dnmt3b was conducive to improving the efficiency of iPSC generation, while the ectopic expression of Dnmt3a and Dnmt3b significantly inhibited reprogramming. Obviously, high expression of Dnmt3a and Dnmt3b is a critical barrier in the reprogramming process, especially at the early stage. However, conditional Dnmt3a or Dnmt3b knockout ES cells fail to differentiate into three germ layers [22]. Dnmt3a- and Dnmt3b-deficient iPSCs also showed restricted developmental potential [21], indicating that Dnmt3a and Dnmt3b might be required for the achievement of full pluripotency. Thus, there must be some unrevealed molecules or mechanisms that strictly control the dynamic expression of Dnmt3a and Dnmt3b during the generation of fully pluripotent iPSCs. We found that the expression of Dnmt3a and Dnmt3b showed a significant inverse correlation with miR-29b expression during reprogramming, and target gene analysis also demonstrated that Dnmt3a and Dnmt3b are in vivo targets of miR-29b. Moreover, during OSKM induction, the introduction of miR-29b sponge led to the upregulation of Dnmt3a and Dnmt3b. Collectively, these data suggest that miR-29b might serve as an intrinsic regulator of Dnmt3a and Dnmt3b and a previously unidentified mediator through which the defined factor Sox 2 accurately regulates the dynamic expression of Dnmt3a and Dnmt3b during the reprogramming process.

Mesenchymal-to-epithelial transition (MET) and the transcriptional silencing of the Dlk1-Dio3 region are crucial DNA methylation-related events during cell reprogramming. MET is required for reprogramming from fibroblasts to pluripotent cells $[17,38]$. The key epithelial gene E-cadherin is critically linked to pluripotency in ES cells and is essential for iPSC generation, and its expression is significantly increased after AZA treatment

Figure 6 miR-29b-DNMT signaling is involved in the MET process and in Dlk1-Dio3 region transcription silencing. (A) miR-29b-DNMT signaling promotes MET during the reprogramming process. QRT-PCR was used to evaluate epithelial gene expression in OG-MEFs induced by OSKM factors or by OSKM in combination with miR-29b on the indicated days of reprogramming. (B) Western blotting for E-cadherin in OG-MEFs induced by OSKM factors or OSKM in combination with miR-29b on days 4, 6, and 8 of reprogramming. (C) Bisulfite sequencing of the E-cadherin promoter in OG-MEFs induced by OSKM factors or OSKM in combination with miR-29b on the indicated days $(0,2,4,6$, and 8$)$ of iPSC induction. (D) The expression of E-cadherin and key mesenchymal genes on day 6 in OG-MEFs transduced by OSKM in combination with Dnmt3a, Dn$\mathrm{mt3b}$, shDnmt3a, or shDnmt3b. (E) The expression levels of the genes and miRNAs in the DIk1-Dio3 region in OSKM iPSCs and OSKM+miR-29b-derived iPSC lines. E14 cells were used as the control. (F) Bisulfite sequencing for the Gt/2 DMR in E14 cells, OSKM iPSCs, and OSKM+miR-29b iPSCs. (G) Analyses of Gt12 and miRNA expression in three individual OSKM-iPSC lines after transfection with miR-29b mimics for $72 \mathrm{~h}$. The error bars represent the standard deviation (SD) of three independent experiments. ${ }^{*} P<0.05,{ }^{* *} P<0.01,{ }^{* * *} P<0.001$, two-tailed Student's $t$-test. 
or the siRNA-mediated depletion of Dnmt1, Dnmt3a or Dnmt3b [17, 39-41]. The normal activation of the Dlk1Dio3 imprinted region is positively correlated with the developmental potential of iPSCs $[18,19]$. Dnmt3a and Dnmt3b have also been shown to be responsible for the methylation of most imprinted regions. Analyses of differentially methylated regions (DMR) indicated that aberrant DNA methylation is responsible for the transcriptional silencing of the Dlk1-Dio 3 region in partially reprogrammed pluripotent iPSCs [20, 42]. We found that, through targeting Dnmt3a and Dnmt3b, miR$29 \mathrm{~b}$ enhanced the expression of E-cadherin, Epcam, and Cldn 3 while inhibiting the expression of mesenchymal genes including Snail, Zeb1, and Cdh2 during the reprogramming process. We also found that OSKM + miR29b-derived iPSC lines maintain the active expression of genes (Gt12 and Rian) and miRNAs from the Dlk1Dio3 locus that are critical for developmental potential. Moreover, the application of miR-29b to OSKM-induced iPSCs with low transcriptional activity of the Dlk1-Dio3 locus significantly activates the expression of genes and miRNAs in this imprinted region. These data indicate that Sox2-triggered miR-29b-DNMT signaling plays a crucial role in DNA methylation-related reprogramming events, including the regulation of MET and Dlk1-Dio3 transcription silencing.

Genome-wide epigenetic remodeling is the determinant factor that leads to the distinct regulation of gene expression during cell reprogramming [43]. During iPSC generation, the defined factors Oct4 and Sox 2 are crucial for regulating epigenetic remodeling events, including DNA methylation. Our study not only improves our understanding of how the defined factor Sox 2 triggers cell reprogramming via a specific miRNA-mediated pathway but also sheds light on the regulation of endogenous Dnmt $3 \mathrm{a} / 3 \mathrm{~b}$ expression and the process of achieving full pluripotency. Improvements in our understanding of the precise mechanism by which defined factors trigger epigenetic remodeling will be helpful to guide further research on somatic cell reprogramming and ultimately contribute to the development of cell therapies and clinical applications.

\section{Materials and Methods}

\section{Cell culture}

Oct4::GFP MEFs (OG-MEFs) were derived from transgenic mice (C57BL/6) at E13.5. OG-MEFs were cultured in highglucose DMEM (Hyclone) with 10\% FBS (Biochrom AG). OGMEFs at passage 0-3 were used for iPSC induction. Plat-E cells and feeder cells were maintained in high-glucose DMEM containing $10 \%$ FBS. To maintain Plat-E cells, $1 \mu \mathrm{g} / \mathrm{ml}$ of puromycin (Sigma) and $10 \mu \mathrm{g} / \mathrm{ml}$ of blasticidin S (Sigma) were added to the culture medium. iPSCs and mouse ES cells were maintained in KOSR medium consisting of knockout-DMEM (Gibco) containing 20\% knockout serum replacement (KOSR) (Gibco), $100 \mu \mathrm{M}$ nonessential amino acids (NEAA) (Thermo), $2 \mathrm{mM}$ L-glutamine (Thermo), and $55 \mu \mathrm{M} \beta$-mercaptoethanol (Gibco) with leukemia inhibitory factor (LIF) (Millipore) on $0.1 \%$ gelatin (Sigma)-coated plates. iPSCs were maintained on feeder layers of mitomycin C (Sigma)-treated MEF cells and passaged every two days. MEFs were isolated as described previously [33] and maintained in highglucose DMEM containing 10\% FBS. In this study, we used MEFs within four passages to avoid replicative senescence, as suggested by previous studies [33].

\section{Vectors}

The vectors used for the four transcription factors in this study were pMX-Oct4, Sox2, Klf4, and c-Myc [1]. DNA fragments corresponding to the precursor sequence of miR-29b were amplified by PCR from NIH3T3 genomic DNA and cloned into the BamHI and Sall restriction sites of the retroviral vector pMX. Dnmt3a and Dnmt3b were inserted into the pBabe-puro retrovirus vector. The DNA encoding the Dnmt3a and Dnmt3b shRNA was cloned into retroviral vector pMKO.1. Dnmt3a 3'UTR and Dnmt3b 3'UTR were cloned into the pGL3-Basic vector. Plasmids pGL3-Dnmt3a3'UTRmut and pGL3-Dnmt3b-3'UTRmut were obtained using the QuickChange Lightning Multi Site Directed Mutagenesis Kit (Agilent Technologies Cat\#210515-5). The plasmids pBabeDnmt3a-3'UTRmut and pBabe-Dnmt3b-3'UTRmut were generated by inserting Dnmt3a-3'UTRmut and Dnmt3b-3'UTRmut into the pBabe-puro SalI and EcoRI restriction sites, respectively. The miR-29b promoter with a Sox 2 binding site was cloned into the pGL3-Control vector. All the constructed plasmids were verified by DNA sequencing. All primers are listed in Supplementary information, Table S1.

\section{Retroviral infection}

To generate the retrovirus, on the day before transfection, PlatE cells were seeded at a density of $8 \times 10^{6}$ cells per 100 -mm dish. On the next day, pMX-based retroviral vectors were introduced into Plat-E cells using the Fugene HD transfection reagent (Roche) according to the manufacturer's recommendations. A $20 \mu \mathrm{l}$ aliquot of Fugene HD transfection reagent was diluted in $500 \mu \mathrm{l}$ of OptiDMEM and incubated for $5 \mathrm{~min}$ at room temperature (RT); then, $8 \mu \mathrm{g}$ of plasmid DNA was added to the mixture and incubated for 15 min at RT. After incubation, the DNA/Fugene HD mixture was added dropwise onto the Plat-E cells. The cells were then incubated overnight at $37{ }^{\circ} \mathrm{C}$ with $5 \% \mathrm{CO}_{2}$. At $8 \sim 10 \mathrm{~h}$ after transfection, the medium was replaced with $8 \mathrm{ml}$ of fresh medium, and the virus-containing supernatant was harvested at $48 \mathrm{~h}$ after transfection. The virus-containing supernatants derived from these PlatE cultures were filtered through $0.45 \mu \mathrm{m}$ Millex-HV (Millipore) filters and combined with $4 \mu \mathrm{g} / \mathrm{ml}$ of polybrene (Sigma).

For iPSC induction, OG-MEFs were seeded at a density of 4 $\times 10^{4}$ cells per well in a gelatin-coated 12 -well plate. Virus-containing supernatant was added to the OG-MEFs and centrifuged at $2000 \mathrm{rpm}$; $90 \mathrm{~min}$ later, the virus-containing supernatant was replaced with $1 \mathrm{ml}$ high-glucose DMEM containing 10\% FBS (Hyclone). Two days later, the medium was replaced with KOSR medium plus LIF, $\beta$-mercaptoethanol, L-glutamine, and NEAA, and the cells were maintained in culture until GFP-positive colo- 
nies appeared. The GFP-positive colonies were mechanically isolated and subsequently replated onto feeder cells.

\section{QRT-PCR for marker genes and miRNA expression}

Total RNA was extracted from cells using the TRIzol-A ${ }^{+}$total RNA Extraction Kit (TIANGEN). cDNA synthesis was performed with the TIANScript RT Kit (TIANGEN) according to the manufacturer's instructions in a reaction volume of $20 \mu \mathrm{l}$.

For QRT-PCR, a template equivalent to $20 \mathrm{ng}$ of total RNA was subjected to 40 cycles of quantitative PCR, and the expression levels of the genes of interest were normalized to that of the gapdh gene. The primer sequences used to amplify endogenous Oct4, Sox2, Klf4, c-Myc, and Nanog are listed in Supplementary information, Table S2.

For quantitative miRNA analyses, we used the Bulge-LoopTM miRNA qPCR Primer Set (RiboBio Co. Cat\#MQP-0101) to detect miRNA expression by QRT-PCR with the SYBR Green qPCR Master Mix (TIANGEN). The relative expression level was calculated by the $2^{-\Delta \Delta \mathrm{Ct}}$ method [34] and represents the amount of candidate miRNA expression relative to the same calibrators and U6 as the internal normalized control.

\section{Alkaline phosphatase staining and immunostaining}

AP staining was carried out using the FastRed Alkaline Phosphatase Kit (Sigma) according to the manufacturer's protocol.

For immunostaining, cells were washed twice with PBS and fixed with $4 \%$ paraformaldehyde at room temperature for $20 \mathrm{~min}$. The fixed cells were permeabilized with $0.2 \%$ Triton $\mathrm{X}-100$ for 5 min and then blocked in PBS/10\% FBS for $1 \mathrm{~h}$ at room temperature. The cells were stained with primary antibody (diluted $1: 1000$ in $\mathrm{PBS} / 10 \% \mathrm{FBS}$ ) overnight at $4{ }^{\circ} \mathrm{C}$, washed three times with $\mathrm{PBS} / 10 \% \mathrm{FBS}$, and then stained for $45 \mathrm{~min}$ in the dark at room temperature with a 1:1000 dilution of the secondary antibody. Cells were stained with anti-Oct4 (Santa Cruz), anti-Nanog (Abcam), or anti-SSEA-1 (Santa Cruz) and counterstained with Hoechst33342. Finally, the cells were examined under a fluorescence microscope to capture both phase contrast and fluorescent images.

\section{Embryoid bodies (EBS) formation and in vitro differentia- tion assay of $i P S C s$}

iPS cells were harvested by trypsinization ( $0.25 \%$ trypsin), and aliquots of $5 \times 10^{5}$ cells were transferred to bacterial culture dishes $(60-\mathrm{mm})$ in $5 \mathrm{ml} \mathrm{KOSR}$ medium without LIF to generate EBs. RNA was extracted from the EBs and used for QRT-PCR analyses. The primers used for QRT-PCR are listed in Supplementary information, Table S6.

For immunostaining analyses of the in vitro differentiation of iPSCs, the iPSCs were trypsinized to produce a single cell suspension, and the hanging drop method was used to generate EBs. Each drop contained $1 \times 10^{3}$ iPSCs in $20 \mu \mathrm{KOSR}$ medium without LIF. EBs were cultured in hanging drops for 3 days and were subsequently reseeded onto gelatin-coated 48 -well plates for another 6 days. The primary antibodies and dilutions used were anti-Tuj1 (1:500, Covance), anti-Gata4 (1:1 000, Santa Cruz Biotechnology), and anti-HNF-3 $\beta$ (1:1 000, Santa Cruz Biotechnology).

Teratoma formation and hematoxylin \& eosin (HE) staining To generate teratomas, iPSCs were trypsinized, resuspended at a concentration of $2 \times 10^{6}$ cells $/ 150 \mu$, and then injected into athymic nude mice (NOD-SCID) that were obtained from the National Resource Center of Mutant Mice Model Animal Research Center (NARC), NJU. Mice were checked for tumors every week for 4 weeks. Tumors were harvested and fixed in a formaldehyde solution for $24 \mathrm{~h}$ at room temperature before paraffin embedding, and the resulting tissue sections were submitted to HE staining.

\section{Production of chimeric mice}

Zygotes were isolated from superovulated female ICR mice, and iPS cells (with a C57BL/6 background) were injected into the resulting blastocysts. Chimeras were produced by implanting the injected blastocysts into pseudopregnant ICR mice.

\section{Western blotting}

Cells were lysed in SDS lysis buffer. Equal amounts of cell lysates were separated by SDS-PAGE, blotted onto a nitrocellulose filter membrane (NC), and probed with the following primary antibodies: anti-Dnmt3a (Cell Signaling, 1:1 000), anti-Dnmt3b (Cell Signaling, 1:1 000), anti-E-cadherin (Abcam), anti-Actin (Invitrogen, 1:750), and anti-GAPDH (Sigma, 1:1 000). GAPDH and Actin were used as loading controls. After incubation with the appropriate secondary antibodies, signals were visualized by enhanced chemiluminescence (ECL) (ImageQuant LAS 4000 mini).

\section{Luciferase reporter assay}

NIH3T3 cells grown in 24-well plates were transfected with 50 nM miR-29b mimics (RiboBio Co.), $0.5 \mu \mathrm{g}$ of pGL-3 luciferase reporter vector, and $0.02 \mu \mathrm{g}$ of a control Renilla luciferase vector (pRL-TK; Promega) in the presence of Lipofectamine 2000 (Invitrogen). The Firefly and Renilla luciferase activities in the cell lysates were assayed with a Dual-Luciferase Reporter Assay System (Promega) at $24 \mathrm{~h}$ post-transfection. Mimics of miRNAs were chemically synthesized mature miRNAs. Inhibitors of miRNAs were chemically synthesized single-stranded RNA and antisense oligonucleotides of mature miRNAs. They can function by competing with endogenous miRNAs for RISC incorporation, and thus hinder the target cleavage mediated by miRNAs.

\section{Chromatin immunoprecipitation (ChIP)}

We performed ChIP with normal rabbit IgG (Millipore) and anti-Sox2 (Abcam) antibodies using an EZ ChIP kit (Millipore) according to the manufacturer's protocol. Immunoprecipitated DNA and whole-cell extract DNA were used as templates for PCR with primers around the binding site $(<500 \mathrm{bp})$ and away from the predicted binding site $(>1000 \mathrm{bp})$. The primers used are listed in Supplementary information, Table S7. Fold enrichment was calculated relative to normal rabbit $\mathrm{IgG}$.

PCRs to detect the genomic integration of transgenes and exogenous transgene silencing

Genomic DNA was extracted from iPSCs using a DNA extraction kit (TIANGEN) to detect the genomic integration of exogenous Oct4, Sox2, Klf4, c-Myc, and miR-29b. The primers used to amplify exo-Oct4, Sox2, Klf4, c-Myc, and miR-29b are listed in Supplementary information, Table S3. cDNA was prepared for the analysis of exogenous Oct4, Sox2, Klf4, c-Myc silencing. The primers used for these analyses are listed in Supplementary information, Table S4. 


\section{Promoter methylation analyses}

Promoter $\mathrm{CpG}$ methylation was analyzed by bisulfite PCR. Briefly, genomic DNA was extracted using a genomic DNA extraction kit (TIANGEN). A total of $1 \mu \mathrm{g}$ of DNA was then used for DNA methylation analyses using the bisulfite PCR primers listed in Supplementary information, Table S5. The products were cloned into the pMD19-T vector (Takara) and then sequenced.

\section{Statistical analyses}

The error bars represent the standard deviation (SD) of three independent experiments. ${ }^{*}$, **, and $* * *$ indicate $P<0.05, P<0.01$, and $P<0.001$, respectively (two-tailed Student's $t$-test).

\section{Acknowledgments}

This work was supported by grants from the Ministry of Science and Technology (2011CB965100, 2011DFA30480, 2010CB944900, 2010CB945000, and 2011CBA01100), the National Natural Science Foundation of China (91219305, 31071306, 31101061, 31210103905, 90919028, 31000378, 31171432, and 30971451), the Science and Technology Commission of Shanghai Municipality (11ZR1438500 and 11XD1405300), and the Ministry of Education (IRT1168 and 20110072110039). The work was also supported by the "Chen Guang" project, the Shanghai Municipal Education Commission and Shanghai Education Development Foundation (12CG19) and the Fundamental Research Funds for the Central Universities.

\section{References}

1 Takahashi K, Yamanaka S. Induction of pluripotent stem cells from mouse embryonic and adult fibroblast cultures by defined factors. Cell 2006; 126:663-676.

2 Takahashi K, Tanabe K, Ohnuki M, et al. Induction of pluripotent stem cells from adult human fibroblasts by defined factors. Cell 2007; 131:861-872.

3 Wernig M, Meissner A, Foreman R, et al. In vitro reprogramming of fibroblasts into a pluripotent ES-cell-like state. $\mathrm{Na}$ ture 2007; 448:318-324.

4 Yu J, Vodyanik MA, Smuga-Otto K, et al. Induced pluripotent stem cell lines derived from human somatic cells. Science 2007; 318:1917-1920.

5 Lowry WE, Richter L, Yachechko R, et al. Generation of human induced pluripotent stem cells from dermal fibroblasts. Proc Natl Acad Sci USA 2008; 105:2883-2888.

6 Park IH, Zhao R, West JA, et al. Reprogramming of human somatic cells to pluripotency with defined factors. Nature 2008; 451:141-146.

7 Kang L, Wang JL, Zhang Y, Kou ZH, Gao SR. iPS cells can support full-term development of tetraploid blastocyst-complemented embryos. Cell Stem Cell 2009; 5:135-138.

8 Okita K, Ichisaka T, Yamanaka S. Generation of germlinecompetent induced pluripotent stem cells. Nature 2007; 448:313-317.

9 Zhao XY, Li W, Lv Z, et al. iPS cells produce viable mice through tetraploid complementation. Nature 2009; 461:86-90.

10 Nishikawa S, Goldstein RA, Nierras CR. The promise of human induced pluripotent stem cells for research and therapy. Nat Rev Mol Cell Biol 2008; 9:725-729.
11 Park IH, Arora N, Huo H, et al. Disease-specific induced pluripotent stem cells. Cell 2008; 134:877-886.

12 Huangfu D, Osafune K, Maehr R, et al. Induction of pluripotent stem cells from primary human fibroblasts with only Oct4 and Sox2. Nat Biotechnol 2008; 26:1269-1275.

13 Shi Y, Do JT, Desponts C, Hahm HS, Scholer HR, Ding S. A combined chemical and genetic approach for the generation of induced pluripotent stem cells. Cell Stem Cell 2008; 2:525528 .

14 Mikkelsen TS, Hanna J, Zhang X, et al. Dissecting direct reprogramming through integrative genomic analysis. Nature 2008; 454:49-55.

15 Huangfu D, Maehr R, Guo W, et al. Induction of pluripotent stem cells by defined factors is greatly improved by smallmolecule compounds. Nat Biotechnol 2008; 26:795-797.

16 Shi Y, Desponts C, Do JT, Hahm HS, Scholer HR, Ding S. Induction of pluripotent stem cells from mouse embryonic fibroblasts by Oct 4 and Klf4 with small-molecule compounds. Cell Stem Cell 2008; 3:568-574.

17 Li RH, Liang JL, Ni S, et al. A mesenchymal-to-epithelial transition initiates and is required for the nuclear reprogramming of mouse fibroblasts. Cell Stem Cell 2010; 7:51-63.

18 Liu L, Luo GZ, Yang W, et al. Activation of the imprinted Dlk1-Dio3 region correlates with pluripotency levels of mouse stem cells. J Biol Chem 2010; 285:19483-19490.

19 Li W, Zhao XY, Wan HF, et al. iPS cells generated without cMyc have active Dlk1-Dio3 region and are capable of producing full-term mice through tetraploid complementation. Cell Res 2011; 21:550-553.

20 Stadtfeld M, Apostolou E, Akutsu H, et al. Aberrant silencing of imprinted genes on chromosome $12 \mathrm{qF} 1$ in mouse induced pluripotent stem cells. Nature 2010; 465:175-181.

21 Pawlak M, Jaenisch R. De novo DNA methylation by Dnmt3a and Dnmt3b is dispensable for nuclear reprogramming of somatic cells to a pluripotent state. Genes Dev 2011; 25:10351040 .

22 Jackson M, Krassowska A, Gilbert N, et al. Severe global DNA hypomethylation blocks differentiation and induces histone hyperacetylation in embryonic stem cells. Mol Cell Biol 2004; 24:8862-8871.

23 Rana TM. Illuminating the silence: understanding the structure and function of small RNAs. Nat Rev Mol Cell Biol 2007; 8:23-36.

24 Judson RL, Babiarz JE, Venere M, Blelloch R. Embryonic stem cell-specific microRNAs promote induced pluripotency. Nat Biotechnol 2009; 27:459-461.

25 Anokye-Danso F, Trivedi CM, Juhr D, et al. Highly efficient miRNA-mediated reprogramming of mouse and human somatic cells to pluripotency. Cell Stem Cell 2011; 8:376-388.

26 Miyoshi N, Ishii $\mathrm{H}$, Nagano $\mathrm{H}$, et al. Reprogramming of mouse and human cells to pluripotency using mature microRNAs. Cell Stem Cell 2011; 8:633-638.

27 Marson A, Levine SS, Cole MF, et al. Connecting microRNA genes to the core transcriptional regulatory circuitry of embryonic stem cells. Cell 2008; 134:521-533.

28 Card DA, Hebbar PB, Li L, et al. Oct4/Sox2-regulated miR302 targets cyclin D1 in human embryonic stem cells. Mol Cell Biol 2008; 28:6426-6438.

29 Lin SL, Chang DC, Lin CH, Ying SY, Leu D, Wu DT. Regu- 
lation of somatic cell reprogramming through inducible mir302 expression. Nucleic Acids Res 2010; 39:1054-1065.

30 Garzon R, Liu S, Fabbri M, et al. MicroRNA-29b induces global DNA hypomethylation and tumor suppressor gene reexpression in acute myeloid leukemia by targeting directly DNMT3A and 3B and indirectly DNMT1. Blood 2009; 113:6411-6418.

31 Fabbri M, Garzon R, Cimmino A, et al. MicroRNA-29 family reverts aberrant methylation in lung cancer by targeting DNA methyltransferases 3A and 3B. Proc Natl Acad Sci USA 2007; 104:15805-15810.

32 Takada S, Berezikov E, Choi YL, Yamashita Y, Mano H. Potential role of miR-29b in modulation of Dnmt3a and Dnmt3b expression in primordial germ cells of female mouse embryos. RNA 2009; 15:1507-1514.

33 Takahashi K, Okita K, Nakagawa M, Yamanaka S. Induction of pluripotent stem cells from fibroblast cultures. Nat Protoc 2007; 2:3081-3089.

34 Livak KJ, Schmittgen TD. Analysis of relative gene expression data using real-time quantitative PCR and the 2(-Delta Delta C(T)) Method. Methods 2001; 25:402-408.

35 Silva J, Nichols J, Theunissen TW, et al. Nanog is the gateway to the pluripotent ground state. Cell 2009; 138:722-737.

36 Maherali N, Sridharan R, Xie W, et al. Directly reprogrammed fibroblasts show global epigenetic remodeling and widespread tissue contribution. Cell Stem Cell 2007; 1:55-70.

37 Kato Y, Kaneda M, Hata K, et al. Role of the Dnmt3 family in de novo methylation of imprinted and repetitive sequences during male germ cell development in the mouse. Hum Mol Genet 2007; 16:2272-2280.

38 Samavarchi-Tehrani P, Golipour A, David L, et al. Functional genomics reveals a BMP-driven mesenchymal-to-epithelial transition in the initiation of somatic cell reprogramming. Cell Stem Cell 2010; 7:64-77.

39 Chen TT, Yuan DT, Wei B, et al. E-cadherin-mediated cellcell contact is critical for induced pluripotent stem cell generation. Stem Cells 2010; 28:1315-1325.

40 Kawasaki H, Taira K. Induction of DNA methylation and gene silencing by short interfering RNAs in human cells. $\mathrm{Na}$ ture 2004; 431:211-217.

41 Rahnama F, Thompson B, Steiner M, Shafiei F, Lobie PE, Mitchell MD. Epigenetic regulation of E-cadherin controls endometrial receptivity. Endocrinology 2009; 150:1466-1472.

42 Stadtfeld M, Apostolou E, Ferrari F, et al. Ascorbic acid prevents loss of Dlk1-Dio3 imprinting and facilitates generation of all-iPS cell mice from terminally differentiated B cells. Nat Genet 2012; 44:398-405.

43 Hochedlinger K, Plath K. Epigenetic reprogramming and induced pluripotency. Development 2009; 136:509-523.

(Supplementary information is linked to the online version of the paper on the Cell Research website.) 